\title{
Multivariate Normalization with Symmetric Diffeomorphisms for Multivariate Studies
}

\author{
B.B. Avants, J.T. Duda, H. Zhang, and J.C. Gee \\ Penn Image Computing and Science Laboratory \\ University of Pennsylvania \\ Philadelphia, PA 19104-6389 \\ avant.s@grasp.cis.upenn.edu
}

\begin{abstract}
Current clinical and research neuroimaging protocols acquire images using multiple modalities, for instance, T1, T2, diffusion tensor and cerebral blood flow magnetic resonance images (MRI). These multivariate datasets provide unique and often complementary anatomical and physiological information about the subject of interest. We present a method that uses fused multiple modality (scalar and tensor) datasets to perform intersubject spatial normalization. Our multivariate approach has the potential to eliminate inconsistencies that occur when normalization is performed on each modality separately. Furthermore, the multivariate approach uses a much richer anatomical and physiological image signature to infer image correspondences and perform multivariate statistical tests. In this initial study, we develop the theory for Multivariate Symmetric Normalization (MVSyN), establish its feasibility and discuss preliminary results on a multivariate statistical study of $22 \mathrm{q}$ deletion syndrome.
\end{abstract}

\section{Introduction}

Emerging imaging modalities such as very high resolution MRI (sub-millimeter), diffusion tensor (DT) MRI and cerebral blood flow imaging provide a unique opportunity for computing anatomically and functionally meaningful mappings between subjects. Traditional high-resolution T1 structural images capture gray matter (computational structure) white matter (connective tissue) and cerebrospinal fluid borders. The diffusion tensor imaging modality [1] provides insight into directional white matter organization in the human brain. This information, captured in a tensor, provides a guide to normalization within white matter that is unavailable to T1 MRI. Arterial spin labeling (ASL) perfusion MRI is another emerging quantitative functional magnetic resonance imaging (fMRI) method which directly measures cerebral blood flow (CBF) by using water molecules in inflowing arteries as an endogenous contrast agent [2]3]. Each modality captures complementary attributes of the underlying subject being imaged. Taken together and in the same coordinate system, the set of these images obtained in a single imaging session on the same subject can be considered as a single multivariate (or multi-spectral) image.

N. Ayache, S. Ourselin, A. Maeder (Eds.): MICCAI 2007, Part I, LNCS 4791, pp. $359-366,2007$.

(C) Springer-Verlag Berlin Heidelberg 2007 
No current image registration method systematically addresses the problem of consistently mapping between two multivariate (MV) datasets. For example, clinical research imaging protocols may acquire T1, DT and cerebral blood flow images. In this case, an individual's anatomical-functional system is represented by, at minimum, these three modalities. MV normalization will directly model this correspondence problem on the level of the individual and her associated set of images, as opposed to on single modality image pairs.

Consider an $n$-modality dataset, $\mathbf{I}_{n}=\left\{I_{1}, \cdots, I_{n}\right\}$ and a second dataset, $\mathbf{J}_{n}$, from a different individual or the same individual at a different time. A common approach to mapping between these individuals is to select one image modality as the reference, for example, $I_{1}$, typically a T1 structural image, and to find the image registration between it and $J_{1}$. However, this approach ignores modality dependent distortions and also does not take advantage of the additional information provided by the alternative pictures of the individuals' anatomicalfunctional systems in modalities $2 \rightarrow n$.

There is little related work, in medical image registration, on this topic. However, in 1993, Miller, et al. published likely the first multivariate deformable normalization method 4 . and applied this method to map between a 2D patient and template dataset. Similarly, Park, et al 5] developed a multiple channel elastic DT registration method for simultaneously mapping T2 and fractional anisotropy (FA) images. FA is a scalar value that measures the degree of anisotropy in each tensor voxel and is aligned, inherently, with the T2 modality. The full tensor is not used in Park's normalization. Miller's recent work [6] is more typical of the approach currently used where individuals' datasets are normalized only according to T1 structural images. Alternatively, normalization may be done only on the DT images $7 / 8$. Our goal is to use as much information about an individual as possible to guide the subject to template matching while allowing (Bayesian) weights modelling the uncertainty between modalities to be incorporated into the normalization optimization. This integrative approach, ideal for setting up multivariate studies, is illustrated in figure 1.

Our MV normalization uses the full MV image object as the basis for finding correspondences. This novel model includes intrasubject transformations between the modalities, intersubject intramodality maps and the ability to spatially vary the weighting of the combined MV data. Our hypothesis is that combining information from, for example, DT and T1 will improve the quality of both gray and white matter mappings and improve statistics derived from multivariate population studies. The diffusion data in DT provides information in white matter areas that appear homogeneous in T1. CBF images, on the other hand, give information about the current state of functionally related blood flow throughout the brain and may also contribute to normalization.

We now extend the symmetric normalization method (SyN) 9 to deal properly with MV datasets, yielding MVSyN. We develop the MVSyN theory for three modalities, two scalar and one tensor. The resulting map may be applied consistently to all three modalities. In our associated study, we use the MV symmetric diffeomorphic transformation model to estimate intersubject 
correspondences from fused T1 and diffusion tensor (DT) structural images. We compare results from MVSyN with standard single modality symmetric normalization in terms of the statistical power for a population study. Our preliminary results suggest the novel finding that combining the DT and T1 pictures of neuroanatomy may indeed improve upon structural normalization usually based on T1 structural data alone.

\section{Multivariate Symmetric Normalization}

Symmetric Diffeomorphic Registration. We now summarize the approach to symmetric normalization, but leave major details to external references 9 . Recall that a diffeomorphism is a differentiable map with a differentiable inverse and may be generated by integrating regularized velocity fields, $\boldsymbol{v}(\mathbf{x}, t)$, through an ordinary differential equation, $d \phi(\mathbf{x}, t)=\boldsymbol{v}(\phi(\mathbf{x}, t), t)$. We denote such a map, indexed by a spatial coordinate $\mathbf{x} \in \Omega$ and a "time" $t$, as $\phi: \mathbf{x} \in$ $\Omega \times t \in[0,1] \rightarrow \Omega$. These diffeomorphic maps are constrained to equal the identity at the boundaries of the domain $\Omega$. The usual approach in image registration is to find a map $\phi(\mathbf{x}, 1)$ such that $I\left(\phi_{1}(\mathbf{x}, 1)\right) \approx J$. This parameterization of the problem is asymmetrically optimized with respect to $I$. The symmetric diffeomorphic parameterization instead seeks solutions $\phi_{1}$ and $\phi_{2}$ such that $I\left(\phi_{1}(\mathbf{x}, 0.5)\right) \approx J\left(\phi_{2}(\mathbf{x}, 0.5)\right)$ where the composition $\phi_{2}^{-1}\left(\phi_{1}(\mathbf{x}, 0.5), 0.5\right)$ gives $\phi_{1}(\mathbf{x}, 1)$. This approach eliminates bias towards either $I$ or $J$. For easier reading we write, for instance, $\phi_{1}(0.5)$ for $\phi_{1}(\mathbf{x}, 0.5)$.

Symmetric Diffusion Tensor Diffeomorphic Registration. We extend the scalar SyN technique for image registration to normalize full tensor data. Diffusion tensor data requires special handling because a transformation of the underlying space should preserve the tensor orientation. To date, no symmetric diffeomorphic image registration method works with the full tensor. Few nonrigid DT normalization strategies incorporate reorientation. We will outline our MV and DT methods for both normalization and reorientation with symmetric diffeomorphisms.

Miller, et al gave an algorithm that maps DT images based upon fiber orientation, which requires projecting each tensor (matrix) to a vector quantity, the principal eigenvector of the tensor [7]. We have recently developed a method in which we are able to estimate small deformation optimization of a DT similarity metric while taking into account the reorientation 8]. We detail our method for using this small deformation model to define velocity fields that may be integrated in time, through the MVSyN framework, to define large deformation diffeomorphisms.

First, define a diffusion tensor as $\mathbf{D}_{\mathbf{i}}$, a symmetric, positive definite $3 \times 3$ matrix. The Euclidean distance between tensors gives a similarity measure that takes advantage of the full tensor of information,

$$
\left\|\mathbf{D}_{1}-\mathbf{D}_{2}\right\|_{D T}=\sqrt{\operatorname{Tr}\left(\left(\mathbf{D}_{1}-\mathbf{D}_{2}\right)^{2}\right)} .
$$


This similarity metric is the DT analogy of the intensity difference measure that may be used for $\mathrm{T} 1$ to $\mathrm{T} 1$ structural image registration.

The difficulty, traditionally, in optimizing this similarity measure with respect to a generic deformable transformation is that the deformation affects the tensor values themselves. An analytical method for parameterizing a deformation in terms of a local affine patch [8] allows one to compute analytical derivatives of the similarity measure above with respect to small deformations.

Now consider $I_{D T}$ and $J_{D T}$, two DT images with pixel values $I_{D T}(\mathbf{x})=$ $\mathbf{D}_{1}, J_{D T}(\mathbf{x})=\mathbf{D}_{2}$. Our goal is to compute the similarity between these two diffusion tensor images with respect to a diffeomorphism, $\phi_{1}$ or $\phi_{2}$,

$$
\int_{\Omega}\left\|I_{D T}\left(\phi_{1}(0.5)\right)-J_{D T}\left(\phi_{2}(0.5)\right)\right\|_{D T}^{2} d \Omega
$$

where we assume tensor reorientation (discussed below) is always used when a transformation is composed with DT images. Consider that a diffeomorphism, $\phi(\mathbf{x})$, is, by definition, a locally affine transformation. Then, in a small neighborhood, $N$, about $\mathbf{x}$, we can approximate the diffeomorphism as an affine transformation, $F$, expressed as $Q S(\mathbf{x})) \mathbf{x}+\mathbf{T}(\mathbf{x})$, where $\mathbf{T}$ is the translation, $Q$ is the rotation matrix and $S$ is the pure deformation component of $F$. We are able to deform images $I_{D T}$ and $J_{D T}$ by using the preservation of principle directions (PPD) method, as given in [10]. Therefore, we may write $I_{D T}\left(\phi_{1}(\mathbf{x})\right)=\tilde{I_{D T}}$ and $J_{D T}\left(\phi_{2}(\mathbf{x})\right)=\tilde{J}_{D T}$, regardless of the size of the $\phi_{i}$, as they are guaranteed diffeomorphic and thus locally affine.

Now assume a small deformation of $\tilde{I_{D T}}$ as $\boldsymbol{v}_{1}$ such that we have $\| \tilde{I_{D T}}(\mathbf{x}+$ $\left.\boldsymbol{v}_{1}\right)-\tilde{J_{D T}} \|_{D T}$. Locally, we estimate $\mathbf{x}+\boldsymbol{v}_{1}(\mathbf{x}) \approx(Q S) \mathbf{x}+\mathbf{T}$. We may then compute, at position $\mathbf{x}$, the equation 2 above as,

$$
\| \tilde{I_{D T}}\left((Q S \mathbf{x}+\mathbf{T})-Q \tilde{J_{D T}} Q^{T} \|_{D T}^{2},\right.
$$

where $Q \tilde{J_{D T}} Q^{T}$ accounts for reorientation and $F$ is estimated in $N$, a local neighborhood of $\mathbf{x}$. Here, we use the finite strain model to parameterize the reorientation, while the more accurate $\mathrm{PPD}$ method has been used to deform the tensor images by the $\phi_{i}$. This parameterization of the similarity allows us to take derivatives of the objective function in the space of diffeomorphisms while taking into account the effect of reorientation on the tensor values. Analytical derivatives of equation 3 are in the proper Eulerian reference frame needed for estimating derivatives of the DT similarity criterion in equation 2, This specialized method, along with PPD reorientation, enables us to extend the SyN method to function with both scalar and diffusion tensor data. Other similarity measures, described in [8], may be parameterized in a similar way. The same approach as above is used to estimate the similarity gradient with respect to $J_{D T}$.

MVSyN with T1, DT, CBF. The extension to MV data requires the use of modality-dependent similarity criterion as well as a strategy for sensibly weighting information from each modality in the registration optimization. Define $\mathbf{I}_{3}$ as 
consisting of $\left\{I_{1}=\mathrm{T} 1, I_{2}=\mathrm{CBF}, I_{3}=\mathrm{DT}\right\}$ image modalities, assumed to exist in the same patient space and in the same rigid orientation. Furthermore, define $\Pi_{3}\left(\mathbf{I}_{\mathbf{3}}, \mathbf{J}_{\mathbf{3}}\right)$ as the similarity criterion operating on a MV pair, $\mathbf{I}_{\mathbf{3}}$ and $\mathbf{J}_{\mathbf{3}}$.

We now extend scalar symmetric normalization to deal with MV datasets,

$$
\begin{array}{r}
E_{M V S y N}\left(\mathbf{I}_{3}, \mathbf{J}_{3}\right)= \\
\inf _{\phi_{1}} \inf _{\phi_{2}} \int_{t=0}^{0.5}\left\{\left\|\boldsymbol{v}_{1}(\mathbf{x}, t)\right\|_{L}^{2}+\left\|\boldsymbol{v}_{2}(\mathbf{x}, t)\right\|_{L}^{2}\right\} d t+ \\
\int_{\Omega} \omega \Pi_{3}\left(\mathbf{I}_{\mathbf{3}}, \mathbf{J}_{\mathbf{3}}, 0.5\right) d \Omega, \\
\text { with each } \phi_{i} \text { the solution of: } \\
\phi_{i} / d t=\boldsymbol{v}_{i}\left(\phi_{i}(\mathbf{x}, t), t\right) \text { with } \phi_{i}(\mathbf{x}, 0)=\mathbf{I d} .
\end{array}
$$

Equation 4 retains the transformation models used in symmetric normalization, but also uses additional similarity metrics, contained in $\Pi_{3}$, for multiple modality image sets. We define $\Pi_{3}$ as,

$$
\begin{array}{r}
\Pi_{3}\left(\mathbf{I}_{\mathbf{3}}, \mathbf{J}_{\mathbf{3}}, 0.5\right)=\omega_{1}(\mathbf{x})\left|I_{1}\left(\phi_{1}(0.5)\right)-J_{1}\left(\phi_{2}(0.5)\right)\right|^{2}+ \\
\left.\omega_{2}(\mathbf{x}) M I\left(I_{2},\left(\phi_{1}(0.5)\right), J_{2}\left(\phi_{2}(0.5)\right)\right)+\omega_{3}(\mathbf{x}) \| I_{3}\left(\phi_{1}(0.5)\right)-J_{3}\left(\phi_{2}(0.5)\right)\right) \|_{D T}^{2}(5
\end{array}
$$

where $M I$ is the mutual information (defined in the usual way) and the $\omega_{i}$ weights each similarity term as a function of the domain. If this term is optimized, we write $\mathbf{I}_{3}\left(\phi_{1}(0.5)\right) \approx \mathbf{J}_{3}\left(\phi_{2}(0.5)\right)$. Our previous work in curve matching [1] showed that weights which vary across the spatial domain allow results that may be superior to results found with constant weighting terms. A reasonable choice for weighting functions will depend upon the relative signal of the input images; for T1 and CBF, levels that are above noise; for DT, the weight may be higher in regions where the fractional anisotropy is strong enough to indicate the presence of white matter. The sum of the weighting functions will be constrained to add to one. We will also normalize each similarity derivative such that its maximum displacement, before weighting, is on the order of a single voxel. This latter step aids in making a fair combination of the different gradients.

Note that the MVSyN registration algorithm involves, qualitatively, taking derivatives of the objective function $\Pi_{3}$ and then regularizing in both space and time. Our approach to regularization uses a finite horizon temporal discretization and the full voxel resolution in the spatial domain. Therefore, the critical gradients which we must compute are the derivatives of $\Pi_{3}$ with respect to the current position of the diffeomorphism. Regularizing this derivative gives an estimate of the velocity. The gradients of $M I$ may be found in [12, for the DT norm in [8].

\section{Results}

We now study a MV dataset containing both DT and structural T1 pediatric neuroimages, as described in [13, and containing 11 controls and 16 age-matched 


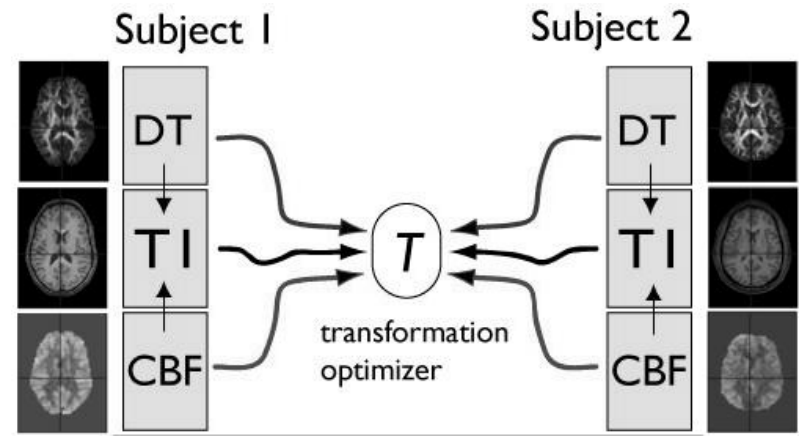

Fig. 1. Two integrative MV datasets of individual anatomical structure and function and the mapping between the MV datasets via transformation, T. Our methods are able to leverage all modalities (T1 structural, DT and cerebral blood flow) to guide the computation of $T$ with maximal subject information. Therefore, in this case, there will be three intramodality inputs for determining $T$. The CBF and DT images are initially mapped into the structural coordinate system, as it is typically of highest resolution and has features shared by each modality. All mappings are diffeomorphic. Curved arrows indicate intersubject transformations while straight arrows indicate intrasubject transformations.

subjects suffering from $22 q$ deletion syndrome. This genetic defect causes a variety of effects, the most prominent of which is brain matter loss and enlarged ventricles. We also chose this dataset because it has been analyzed previously with other methods [13] and contains a known population difference showing reductions in the gray matter of $22 q$ subjects relative to controls.

Preprocessing, before normalization, involved mapping each subject's DT image into the T1 anatomical space by an affine registration. Affine registration was used to accommodate for modality-dependent distortion. The T1 structural images were also skull-stripped. We then select a control image as a template and normalize the full dataset to this template by optimizing equation 4 . For this preliminary comparison, we choose constant weighting functions $\omega_{1}=0.5$, $\omega_{2}=0.0$ and $\omega_{3}=0.5$. The results of an example normalization, along with multivariate group difference statistics on the fractional anisotropy (FA) and Jacobian, are shown in figure 2. The statistics were assessed with Hotelling's $T^{2}$ distribution where the variables were, at each voxel, the value of the normalized FA and the log-Jacobian of the diffeomorphic map to the template space. The Jacobian estimates local structure sizes. The log-Jacobian is used as it has a symmetric distribution around zero. Therefore, our multivariate study assesses the probability of a control-patient difference in both FA and local structure sizes. For comparative purposes, we also performed the same study with SyN, using the T1 structural images alone to guide the mapping. This involved using MVSyN but with $\omega_{2}$ and $\omega_{3}$ set to zero. Both FA and Jacobian images were used for the MVSyN and SyN multivariate comparison.

The significance of our results were assessed with cluster-size based permutation tests. Permutation tests provide a non-parametric method for examining 


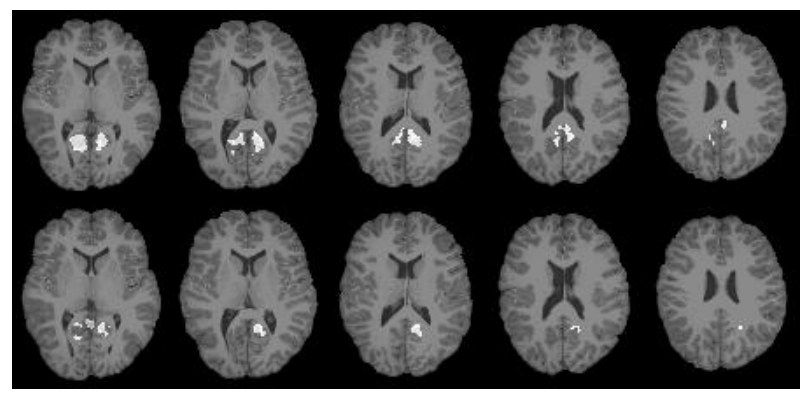

Fig. 2. The most significant cluster generated by MVSyN normalization is shown overlaid on the template in the top row. The cluster shows the areas where gray matter and FA are significantly larger in controls compared to subjects (non-parametric cluster level significance at $p<0.005$ ). The bottom row shows the largest cluster generated by SyN normalization (1003 voxels, significant at $p<0.025$. The MVSyN cluster is larger and also more bilateral. It is interesting that including DT information in the normalization increases the significance of a gray-matter focused result.

the random chance of finding results similar to those in one's true population. The procedure involves selecting a $\mathrm{T}$ or $p$-value threshold and then collecting the histogram of cluster sizes over hundreds or thousands of permutations of the dataset. We chose a threshold of $p<0.001$ to generate clusters at each of 1000 permutations.

Our comparison found that MVSyN produced stronger MV statistics for this study. A cluster size of 938 voxels was significant at a level of $p<0.05$ for MVSyN. A cluster size of 1113 voxels was significant for SyN at $p<0.05$. These results, and the major cluster found by both methods, are shown in figure 2. The largest above threshold cluster, generated by MVSyN, was 1643 voxels. SyN's corresponding cluster was 1003 voxels in size. Both clusters show gray matter loss in 22q subjects relative to controls, as expected, as well as a difference in the FA. By examining the univariate statistics, we saw that the majority of this effect was due to volumetric, rather than FA, differences. Our finding, here, is consistent with previous studies 13, although our statistical threshold is more conservative.

\section{Discussion}

The most common approach for multiple modality studies is to perform normalization with respect to structural images alone. MVSyN absorbs this model while permitting much greater flexibility through parameter selection and using full MV datasets. We showed that the MVSyN model also improves the statistical significance of a MV statistical study on a known patient population. We also note that univariate statistics on the Jacobian values generated by $\mathrm{SyN}$ and MVSyN show the same trend as the multivariate statistics.

We believe that MVSyN provides an ideal complement to multivariate datasets currently being collected and will improve normalization-based studies. However, 
many issues remain, including parameter selection, similarity metric choice and evaluation. One pressing issue not explicitly dealt with here is the possibility of non-rigid deformation in the intrasubject T1 to DT or CBF to T1 mapping. Note that, if necessary, one may introduce explicit DT distortion correction models. Furthermore, development in parallel MR imaging are reducing the amount of distortion present in DT. For these reasons, and given our encouraging preliminary results, we believe MVSyN constitutes a valuable contribution to normalization methodology.

\section{References}

1. Basser, P J, Mattiello, J., Le Bihan, D.: MR diffusion tensor specstroscopy and imaging. Biophys. J. 66, 259-267 (1994)

2. Wang, J., Licht, D.J., Jahng, G.H., Liu, C.S., Rabin, J.T., Haselgrove, J.C.: Pediatric perfusion imaging using pulsed arterial spin labeling. J. Mag. Reson. Imag. 18, 404-413 (2003)

3. Wang, J., Aguirre, G., Kimberg, D., Roc, A.C., Li, L., Detre, J.: Arterial spin labeling perfusion fmri with very low task frequency. Magn. Reson. Med. 49, 796$802(2003)$

4. Miller, M.I., Christensen, G.E., Amit, Y., Grenander, U.: Mathematical textbook of deformable neuroanatomies. Proc. Natl. Acad. Sci (USA) 90, 11944-11948 (1993)

5. Park, H.-J., Kubicki, M., Shenton, M E, et al.: Spatial normalization of diffusion tensor MRI using multiple channels. Neuroimage 20, 1995-2009 (2003)

6. Miller, M.I., Beg, M.F., Ceritoglu, C., Stark, C.: Increasing the power of functional maps of the medial temporal lobe by using large deformation diffeomorphic metric mapping. PNAS 102, 9685-9690 (2005)

7. Yan, C., Miller, M.I., Winslow, R.L., Younes, L.: Large deformation diffeomorphic metric mapping of vector fields. tmi 24, 1216-1230 (2005)

8. Zhang, H., Yushkevich, P.A., Alexander, D.C., Gee, J.C.: Deformable registration of diffusion tensor MR images with explicit orientation optimization. In: Duncan, J.S., Gerig, G. (eds.) MICCAI 2005. LNCS, vol. 3749, Springer, Heidelberg (2005)

9. Avants, B., Grossman, M., Gee, J.C.: Symmetric diffeomorphic image registration: Evaluating automated labeling of elderly and neurodegenerative cortex. Medical Image Analysis (in press online, 2007)

10. Alexander, D.C., Pierpaoli, C., Basser, P.J., Gee, J.C.: Spatial transformations of diffusion tensor magnetic resonance images. IEEE Trans. Med. Imaging 20, 11311139 (2001)

11. Avants, B., Gee, J.C.: Formulation and evaluation of variational curve matching with prior constraints. In: Gee, J.C., Maintz, J.B.A., Vannier, M.W. (eds.) Biomedical Image Registration, pp. 21-30. Springer, Heidelberg (2003)

12. Hermosillo, G., Chefd'Hotel, C., Faugeras, O.: A variational approach to multimodal image matching. Intl. J. Comp. Vis. 50, 329-343 (2002)

13. Simon, T., Ding, L., Bish, J., McDonald-McGinn, D., Zackai, E., Gee, J.C.: Volumetric, connective, and morphologic changes in the brains of children with chromosome 22q11.2 deletion syndrome: an integrative study. Neuroimage 25, 169-180 (2005) 